\title{
Exact solution of two-dimensional MHD boundary layer flow over a semi-infinite flat plate
}

\author{
Ramesh B. Kudenatti ${ }^{\text {a, } *}$, Shreenivas R. Kirsur ${ }^{b}$, L.N. Achala ${ }^{b}$, N.M. Bujurke ${ }^{c}$

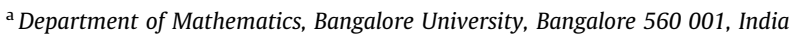 \\ ${ }^{\mathrm{b}}$ P.G. Department of Mathematics and Research Centre in Applied Mathematics, MES College, Malleswaram, Bangalore 560 003, India \\ ${ }^{\mathrm{c}}$ Department of Mathematics, Karnatak University, Dharwad 580 003, India
}

\section{A R T I C L E I N F O}

\section{Article history:}

Received 13 September 2012

Accepted 25 September 2012

Available online 23 October 2012

\section{Keywords:}

Boundary layer equations

MHD

Exact solution

Asymptotic solutions

Dirichlet series

\begin{abstract}
A B S T R A C T
In the present paper, an exact solution for the two-dimensional boundary layer viscous flow over a semi-infinite flat plate in the presence of magnetic field is given. Generalized similarity transformations are used to convert the governing boundary layer equations into a third order nonlinear differential equation which is the famous MHD Falkner-Skan equation. This equation contains three flow parameters: the stream-wise pressure gradient $(\beta)$, the magnetic parameter $(M)$, and the boundary stretch parameter $(\lambda)$. Closed-form analytical solution is obtained for $\beta=-1$ and $M=0$ in terms of error and exponential functions which is modified to obtain an exact solution for general values of $\beta$ and $M$. We also obtain asymptotic analyses of the MHD Falkner-Skan equation in the limit of large $\eta$ and $\lambda$. The results obtained are compared with the direct numerical solution of the full boundary layer equation, and found that results are remarkably in good agreement between the solutions. The derived quantities such as velocity profiles and skin friction coefficient are presented. The physical significance of the flow parameters are also discussed in detail.
\end{abstract}

(c) 2012 Elsevier B.V. All rights reserved.

\section{Introduction}

The study of two-dimensional magnetohydrodynamic boundary layer flows of a viscous fluid have attracted large number of researchers during the last decade because of their increasing applications in engineering and technology, such as MHD power generators, MHD flow meters and pumps, polymer industry, spinning of filaments, etc. In industrial applications, when sheets or filaments are subjected to cooling through quiescent fluid, these essentially get stretched, but this cooling of the sheets could be managed by applying the magnetic field, so that we can expect the final products with desired characteristics. Because of such important applications, many investigators have modeled the behavior of a MHD boundary layer flow. Kumaran et al. [1] have investigated MHD boundary layer flow of an electrically conducting fluid past a quadratically stretching sheet, and have shown that magnetic field makes the streamlines steeper which results the boundary layer thinner. Joneidi et al. [2] undertook the study of heat and mass transfer of a viscous and electrically conducting fluid in the presence of the magnetic field, and have shown that magnetic field decreases the velocity profiles. Su and Zheng [3] have used the differential transform method to investigate the MHD Falkner-Skan flow over a permeable plate in the presence of a transverse magnetic field, and have discussed the effects of various physical parameters on the boundary layer flow. Exploring a Chebyshev pseudospectral differentiation matrix method, Guedda et al. [4] have investigated two-dimensional mixed convection boundary layer flow over a flat plate which is embedded in porous medium in the presence of applied magnetic field.

\footnotetext{
* Corresponding author. Tel.: +91 080 22961424; fax: +91 8022219714.

E-mail address: ramesh@bub.ernet.in (R.B. Kudenatti).
} 
However, the fundamental equations for MHD flow over a semi-infinite flat plate are essentially complicated and highly non-linear in nature. Most of the studies on MHD boundary layers in the literature have been using numerical (shooting methods or Keller-box finite difference methods, etc.) methods for their solutions. Although few almost approximate analytical methods have been reported for MHD Falkner-Skan equation, but no exact solutions are available. Abbasbandy and Hayat [5] have used recently developed homotopy analysis method for the solution of MHD Falkner-Skan equation for all physical parameters. This study has been extended by Hendi and Hussain [6] by including more general boundary conditions and solved again by homotopy analysis method. Parand et al. [7] have developed Hermite functions pseudospectral method for the solution of MHD Falkner-Skan equation for various values of pressure gradient and magnetic parameters. In any case they have compared their results with that of direct numerical solution (DNS) of the problem. Nevertheless, no exact solution is reported in the literature. The present paper contributes to this line of enquiry by giving exact solution of the MHD Falkner-Skan equation for all involved parameters.

In this present paper, we have made an attempt to give an exact solution of MHD Falkner-Skan equation for general value of $\beta$, Hartman number $M$ and boundary stretch parameter $\lambda$. This has been achieved through the method pioneered by Sachdev et al. [8] for solution of the Falkner-Skan equation. We obtain a closed-form solution of the MHD Falkner-Skan equation for $\beta=-1$ and $M=0$ which is expressed in the form of error and exponential functions. We exploit the nature of the known closed-form solution for $\beta=-1$ and $M=0$. This solution can be rewritten, for convenience, in the form

$$
f(\eta)=\eta+\delta-\frac{\delta}{G(\eta)}
$$

where $\delta$ is the displacement thickness of the boundary layer, and the new stream function $G(\eta)$ shall be defined later in the paper. The above form has been utilized to obtain the exact solution for all values of $\beta$ and $M$. Exploring the above form, Kudenatti and Awati [9] and Kudenatti [10] have given an exact solution of the Falkner-Skan flow over a semi-infinite flat plate in absence of the magnetic field, and their results are remarkably agreeing to the DNS of the problem.

Rest of the paper is organized as follows. The two-dimensional MHD laminar boundary layer equations and corresponding similarity transformations are given in Section 2, and derived MHD Falkner-Skan equation with relevant boundary conditions. However, this derivation is just an extension of Sachdev et al. [8] for including the magnetic field, but to make this paper self-contained, it is derived in the present paper. An exact solution of the MHD Falkner-Skan equation for $\beta=-1$ and $M=0$ is also given in Section 2. In Section 3, we give an exact solution of the MHD Falkner-Skan equation for all general values of pressure gradient parameter $\beta$ and magnetic number $M$. In Section 4 , two asymptotic solutions for large $\lambda$ and large $\eta$ are also given. In the former, the MHD Falkner-Skan equation can be exactly linearized about $f^{\prime}(\eta) \rightarrow 1$ as $\eta \rightarrow \infty$. In the case of latter, we use different transformation for MHD Falkner-Skan equation wherein it turns into other family of boundary layer equation, we use the Dirichlet series; highly efficient technique, for its solution. In Section 5, we compare the results obtained by our exact method with that of the DNS. We also compare the results of Dirichlet series method with that of the DNS. The concluding section summarizes the major results of the present study.

\section{Formulation of the problem}

The model consists of the MHD laminar boundary layer flow of a viscous and incompressible fluid over a flat plate moving with a constant velocity $U_{w}(x)$ in the presence of magnetic field $\check{B}(x)$. Strength of induced electric field due to polarization of charges is negligible along with Reynolds number is also too small. The $x$-axis is taken along the boundary layer flow, and $y$ axis is normal to it. Under these approximations, the governing boundary layer equations are given by

$$
\begin{aligned}
& \frac{\partial u}{\partial x}+\frac{\partial v}{\partial y}=0 \\
& u \frac{\partial u}{\partial x}+v \frac{\partial u}{\partial y}=U(x) \frac{\partial U(x)}{\partial x}+v \frac{\partial^{2} u}{\partial y^{2}}-\frac{\sigma B^{2}(x)}{\rho}(u-U(x))
\end{aligned}
$$

where $u$ and $v$ are the velocity components in the $x$ and $y$ directions, $v$ is the kinematic viscosity of the fluid, $\sigma$ is the electrical conductivity, $\rho$ is the fluid density, and the magnetic field is given by $B(x)=\breve{B} x^{(m-1) / 2}, U(x)$ is the velocity at the edge of the boundary layer which obeys the power law relation $U(x)=U_{\infty} x^{m}$, where $x$ is the distance measured from the onset of the boundary layer, $U_{\infty}$ and $m$ are constants. The relevant boundary conditions for the above model are

$$
\begin{aligned}
& \text { at } y=0: \quad u=U_{w}(x), \quad v=0, \quad \text { and } \\
& \text { as } \frac{y}{\delta} \rightarrow \infty: \quad u \rightarrow U(x),
\end{aligned}
$$

where $U_{w}(x)$ is the stretching surface velocity which obeys the power-law relation $U_{w}(x)=U_{0 w} x^{m}$. Introducing the streamfunction $\psi(x, y)$ as

$$
u=\frac{\partial \psi}{\partial y}, \quad v=-\frac{\partial \psi}{\partial x},
$$


where the similarity transformation $\psi(x, y)$ is given by

$$
\psi=\sqrt{\frac{2 v x U(x)}{1+m}} f(\eta), \quad \eta=\sqrt{\frac{(1+m) U(x)}{2 v x}} y, \quad \beta=\frac{2 m}{m+1}
$$

into the above nonlinear system (2)-(4), we get the MHD Falkner-Skan equation as

$$
f^{\prime \prime \prime}(\eta)+f(\eta) f^{\prime \prime}(\eta)+\beta\left(1-f^{\prime 2}(\eta)\right)-M^{2}\left(f^{\prime}(\eta)-1\right)=0, \quad{ }^{\prime}=\frac{d}{d \eta}
$$

with the boundary conditions

$$
f(0)=0, \quad f^{\prime}(0)=-\lambda, \quad f^{\prime}(+\infty)=1 .
$$

Here $f(\eta)$ is the non-dimensional stream-function, and $\eta$ is a new similarity variable, and $\lambda\left(=-\frac{U_{w}}{U_{\infty}}\right)$ is the ratio of free stream velocity to boundary velocity, $\lambda>0$ and $\lambda<0$ correspond to moving plate in opposite and in the same direction to the freestream velocity, whereas $\lambda=0$ is the case for fixed plate, $\beta$ is the stream-wise pressure gradient parameter, $\beta>0$ represents favorable pressure gradient, and $\beta<0$ is the adverse pressure gradient, whereas $\beta=0$ is the Blasius flow over a flat plate. The parameter $M\left(=\sqrt{\frac{2 \sigma}{\rho U_{\infty}(m+1)}} B\right)$ is the magnetic (Hartmann number) parameter which is the ratio of electromagnetic force to the viscous force.

Integration of (6) and (7) with $\beta=-1$ and $M=0$ gives the Riccati type equation:

$$
f^{\prime}(\eta)+\frac{f^{2}(\eta)}{2}=\frac{\eta^{2}}{2}+\delta \eta-\lambda
$$

where $\delta=f^{\prime \prime}(0)$. The solution of (8) is given by

$$
f(\eta)=\eta+\delta-\frac{\delta e^{-\left(\frac{\eta^{2}}{2}+\delta \eta\right)}}{1-\sqrt{\frac{\pi}{2}} \frac{\delta}{2} e^{\left(\frac{\delta^{2}}{2}\right)}\left(\operatorname{erf}\left(\frac{\eta+\delta}{\sqrt{2}}\right)-\operatorname{erf}\left(\frac{\delta}{\sqrt{2}}\right)\right)}
$$

provided $\delta= \pm \sqrt{-2(1+\lambda)}$. The axial velocity gradient at the wall is given by

$$
f^{\prime \prime}(0)=\delta= \pm \sqrt{-2(1+\lambda)}
$$

From Eq. (10), it follows that, the MHD Falkner-Skan equation with $\beta=-1$, and $M=0$ exhibits dual solutions for $\lambda<0$ and no solution for $\lambda>-1$. For $\lambda=1$, a trivial solution of the Falkner-Skan equation is $f(\eta)=\eta$, which seems to demarcate the solution nature. The above solution (9) can be taken directly from Sachdev et al. [8], but, for ease of completeness, and further use in the present paper, it has been given again.

This present paper devices a method that helps in obtaining a new exact solution of the MHD Falkner-Skan equation (6) and (7) for general values of $\beta$ and $M$. The solution recovers the known closed-form solution as a special case.

\section{Exact solution for general $\beta$ and $M$}

Note that an exact solution of the MHD Falkner-Skan system (6) and (7) for $\beta=-1$ and $M=0$ has been already obtained that can be rewritten, for convenience, in modified form

$$
f(\eta)=\eta+\delta-\frac{\delta}{G(\eta)}
$$

where $G(\eta)$ is given by

$$
G(\eta)=\left(e^{-\frac{\delta^{2}}{2}}+\sqrt{\frac{\pi}{2}} \frac{\delta}{2} \operatorname{erf}\left(\frac{\delta}{\sqrt{2}}\right)\right) e^{\frac{(\eta+\delta)^{2}}{2}}-\sqrt{\frac{\pi}{2}} \frac{\delta}{2} e^{\frac{(\eta+\delta)^{2}}{2}} \operatorname{erf}\left(\frac{\eta+\delta}{2}\right) .
$$

The stream function $G(\eta)$ in Eq. (11) easily generalizes the solution of the system (6) and (7) for all values of $\beta$ and $M$. Substituting the form (11) into the system (6) and (7), we get another nonlinear ordinary differential equation in $G(\eta)$ which contains the parameters $\beta$ and $M$, as

$$
G^{2} G^{\prime \prime \prime}-\left(6 G^{\prime}+\delta-(\eta+\delta) G\right) G G^{\prime \prime}-\left(2 \beta+M^{2}\right) G^{2} G^{\prime}+(\delta(2-\beta)-2(\eta+\delta) G) G^{2}+6 G^{\prime 3}=0,
$$

and the boundary conditions become

$$
G(0)=1, \quad G^{\prime}(0)=\frac{\delta}{2}, \quad G(+\infty)=\infty .
$$

The exact analytical solution of (13) and (14) for $\beta=-1$ and $M=0$ is given by Eq. (12). The error and exponential functions in Eq. (12) are entire functions. These functions may be expanded in Taylor series about $\eta=0$ which have an infinite radius 
of convergence. Therefore, the series representation of the above solution for $\beta=-1$ and $M=0$ becomes main clue for further similar analysis for other values $\beta$ and $M$. Thus, motivated by the series representation of (12), we let

$$
G(\eta)=\sum_{n=0}^{\infty} a_{n} \eta^{n}
$$

for general $\beta$ and $M$. Substituting (15) into (13) and equating the coefficients of $\eta^{n}$ to zero, we get

$$
\begin{aligned}
& a_{0}=1, \quad a_{1}=\frac{\delta}{2}, \\
& a_{3}=\frac{\delta}{24}\left(2 M^{2}+4 \beta+(-3+\beta) \delta^{2}+24 a_{2}\right), \\
& a_{4}=\frac{1}{96}\left(2\left(1+3 M^{2}+6 \beta\right) \delta^{2}+(-5+2 \beta) \delta^{4}+4 a_{2}\left(-2+2 M^{2}+5 \delta^{2}+2 \beta\left(2+\delta^{2}\right)+24 a 2\right)\right)
\end{aligned}
$$

and in general the recurrence relation is given by

$$
\begin{aligned}
a_{n+3}= & \frac{-1}{(n+1)(n+2)(n+3)}\left(\sum_{m=0}^{n-1} \sum_{k=0}^{n-m}(m+1)(m+2)(m+3) a_{k} a_{n-m-k} a_{m+3}\right. \\
& +\sum_{m=0}^{n}(-\delta)(m+1)\left((m+2) a_{n-m} a_{m+2}-(2-\beta)(n-m+1) a_{m+1} a_{n-m+1}\right) \\
& -\sum_{m=0}^{n} \sum_{k=0}^{m}(k+1)(m-k+1)\left(2 \delta a_{n-m}-6(n-m+1) a_{n-m+1}\right) a_{k+1} a_{m-k+1} \\
& +\sum_{m=0}^{n} \sum_{k=0}^{n-m}\left((m+1)\left(m a_{m+1}+\delta(m+2) a_{m+2}-2 \beta a_{m+1}-M^{2} a_{m+1}\right) a_{k} a_{n-m-k}\right. \\
& \left.\left.-(k+1)\left(6(m+1)(m+2) a_{m+2}+2(n-m-k) a_{m}\right) a_{k+1} a_{n-m-k}\right)\right)
\end{aligned}
$$

for $n=1,2,3, \ldots$. It is obvious from the above coefficients and the recurrence relation that all coefficients $a_{n}$ can be expressed in terms of two known parameters $\beta$ and $M$, and two free parameters $a_{2}$ and $\delta$. The constant $a_{2}$ which characterizes the coefficient of skin friction remains unknown because of the last condition at (14) which must be found such that the derivative boundary condition at far distance is satisfied. Thus, the unknown $a_{2}$ is related to $f^{\prime \prime}(0)$ through the following

$$
a_{2}=\frac{\left(f^{\prime \prime}(0)+\frac{\delta^{3}}{2}\right)}{2 \delta} \text {. }
$$

It is worth mentioning here that we need to find the unknown $a_{2}$ of the series (15) or $f^{\prime \prime}(0)$ of the MHD Falkner-Skan system (6) and (7). On the other hand, the other unknown constant $\delta$ expressed in Eq. (10) was known only for $\beta=-1$ and $M=0$. To find this $\delta$ for other values of $\beta$ and $M$, we patch the series expansion of (12) with that of general series (15) for $\beta=-1$ and $M=0$, surprisingly we get the same constant: $\delta=\sqrt{-2(1+\lambda)}$. The other constant $a_{2}$ or $f^{\prime \prime}(0)$ can be determined from the following integral relation:

$$
\int_{0}^{\infty}\left(f^{\prime}(\eta)-f^{\prime 2}(\eta)\right) d \eta+\beta \int_{0}^{\infty}\left(1-f^{\prime 2}(\eta)\right) d \eta-M^{2} \int_{0}^{\infty}\left(f^{\prime}(\eta)-1\right) d \eta=f^{\prime \prime}(0) .
$$

Both left and right hand side of the above relation (19) involves $f^{\prime \prime}(0)$, it is natural solve for $f^{\prime \prime}(0)$ iteratively with suitable initial condition. Furthermore, in order to effectively describe the iteration method used to determine the skin friction coefficient $f^{\prime \prime}(0)$, we rewrite for convenience the above integral relation as

$$
\int_{0}^{\eta_{\max }}\left(f^{\prime}(\eta)-f^{\prime 2}(\eta)\right) d \eta+\beta \int_{0}^{\eta_{\max }}\left(1-f^{\prime 2}(\eta)\right) d \eta-M^{2} \int_{0}^{\eta_{\max }}\left(f^{\prime}(\eta)-1\right) d \eta=f^{\prime \prime}(0),
$$

where $\eta_{\max }$ is the maximum value of $\eta$ at the edge of the boundary layer. The solution of the above asymptotic integral relation is too complicated by the fact that the boundary condition is specified at infinity. Therefore, in our computations, 'infinity' is numerically approximated by the large value of independent variable (i.e. $\eta_{\max }$ ). Giving too small numerical value for $\eta_{\max }$ would not assure a uniformly valid convergent solution. And selecting too large value for $\eta_{\max }$ results either in asymptotic divergent series or in slow convergence of the series. Hence, a method must be modified to logically estimate the value of $\eta_{\max }$ to get $f^{\prime \prime}(0)$ up to required accuracy. During iteration operation, the initial approximation of $f^{\prime \prime}(0)$ is taken from the known exact analytical solution (9) for all other parameter $\beta$ and $M$ which serves a good initial estimate for $f^{\prime \prime}(0)$, and also it ensures the fast convergence. For small value of $\eta_{\max }$, the series is well behaved and can be integrated. So, in the process, once $f^{\prime \prime}(0)$ is assumed, and the Pade' approximants which extend the region of validity of convergence, are used to sum the series, the integral relation can be numerically integrated using Simpson's $\frac{1}{3} \mathrm{rd}$ technique without any difficulty to determine a nearly correct value of $f^{\prime \prime}(0)$. With fewer iterations, $f^{\prime \prime}(0)$ can be obtained up to any desired accuracy. All of our simulations 
indicate that the form (11) through series (15) gives convergent solution via Pade approximants. The results thus obtained for various values of $\beta$ and $M$ by the present method are seen to agree with those produced by the DNS of the MHD FalknerSkan equation. The results obtained by the method described above have been presented in terms of velocity profiles in figures, and of the skin friction coefficient in tables.

\section{Asymptotic solutions}

\subsection{Asymptotics for $\lambda \rightarrow \infty$}

In this section, we investigate the behavior of large $\lambda$ asymptotics of the MHD Falkner-Skan equation. Interestingly, under the transformations $f(\eta)=\lambda^{1 / 2} H(z), \eta=\lambda^{-1 / 2} z$ and $M=\lambda^{1 / 2} M^{*}$, the systems (6) and (7) convert into another version of boundary layer equation:

$$
H^{\prime \prime \prime}(z)+H(z) H^{\prime \prime}(z)-\beta H^{\prime 2}(z)-M^{* 2} H^{\prime}(z)=0,
$$

and corresponding boundary conditions become

$$
H(0)=0, \quad H^{\prime}(0)=-1, \quad H^{\prime}(\infty)=0
$$

as $\lambda \rightarrow+\infty$. Now primes denote differentiation with respect to $z$. Note that two systems (6), (7) and (21), (22) look mathematically similar, but their physical significance is entirely different. This aspect can be made clear later in the paper. The systems (21) and (22) describe a viscous boundary layer flow due to constant shrinking sheet where the velocity on the boundary is towards the origin or a fixed point, and this is relatively new in the boundary layer theory. The problems of shrinking sheet have been extended to discuss the various aspects of flow and heat transfer phenomenon with and without MHD by large number of investigators. Using numerical techniques, Fang [11] investigated the boundary layer flow over a continuously shrinking sheet with a power-law surface velocity, and observed that the velocity overshoot near edge of the boundary layer for certain solution branches. Bachok et al. [12] have numerically illustrated an unsteady viscous flow over a continuously permeable shrinking surface, and for a small range of physical parameters, they found dual solutions for shrinking sheet. Sajid and Hayat [13] have used homotopy analysis method to solve MHD two-dimensional and axisymmetric shrinking sheet. Because of the significant applications, the shrinking sheet problem has been studied in different aspects, examples include second-grade fluid (Hayat et al. [14]), sheet with slip in micropolar fluid (Das [15]), unsteady porous sheet with variable viscosity (Nadeem and Awais [16]), MHD heat transfer analysis (Javed et al. [17]), etc. Guedda and Ouahsine [18] have investigated a two-dimensional MHD boundary layer flow over a permeable surfaces and shown that existence of solution that leads to multiple solutions for some model control parameters.

A close examination of the derivative boundary condition at infinity motivates us to look for the Dirichlet series solution which ideally suits for particular boundary condition. This method computes the derived quantities such as velocity profiles and skin friction without much difficulty. Kravnchenko and Yablonskii [19] were the first to adopt the Dirichlet series for solving third order nonlinear boundary value problem wherein derivative boundary condition at infinity is zero. Recently, Kudenatti et al. [20] have used the Dirichlet series to analyze a class of boundary layer equations over nonlinearly stretching surface. All the problems were provided with skin friction values $\left(H^{\prime \prime}(0)\right)$. Exploring the Dirichlet series (different from (23) below) they successfully solved and compared with numerical results and found that results are in agreement. A general discussion of the convergence of the Dirichlet series may be found in Riesz [21]. We choose the base function for the above Eq. (21) in the form

$$
H(z)=\frac{B_{1}}{B_{0}}+6 B_{1} \sum_{n=1}^{\infty} b_{n} B_{2}^{n} e^{-n B_{1} z},
$$

where $B_{0}, B_{1}$ and $B_{2}$ are unknown constants to be determined, and are functions of $\beta$ and $M^{*}$. Note that solution always exists when $B_{1}>0$ and $\left|B_{2}\right|<1$ conditions satisfy. Note that the above base function automatically satisfies the last condition in (22). Substituting function (23) into the boundary value problem (21), we get the following recurrence relation:

$$
\begin{aligned}
& -6 B_{1}^{4} \sum_{n=1}^{\infty} n^{3} b_{n} B_{2}^{n} e^{-n B_{1} z}+6 \frac{B_{1}^{4}}{B_{0}} \sum_{n=1}^{\infty} n^{2} b_{n} B_{2}^{n} e^{-n B_{1} z}+36 B_{1}^{4} \sum_{n=2}^{\infty} \sum_{k=1}^{n-1} k^{2} b_{n-k} b_{k} B_{2}^{n} e^{-n B_{1} z}-36 \beta B_{1}^{4} \sum_{n=2}^{\infty} \sum_{k=1}^{n-1} k(n-k) b_{n-k} b_{k} B_{2}^{n} e^{-n B_{1} z} \\
& +6 M^{* 2} B_{1}^{2} \sum_{n=1}^{\infty} n b_{n} B_{2}^{n} e^{-n B_{1} z}=0 .
\end{aligned}
$$

For $n=1$, the above recurrence relation gives

$$
B_{0}=\frac{B_{1}^{2}}{B_{1}^{2}-M^{* 2}} .
$$

It pointed out from our extensive simulation below that $B_{1} \neq M^{*}$. Therefore, we rewrite the above recurrence relation (24) as 


$$
b_{n}=\frac{6 B_{1}^{2}}{n(n-1)\left(n B_{1}^{2}+M^{* 2}\right)} \sum_{k=2}^{n-1}(k-\beta(n-k)) k b_{k} b_{n-k}
$$

for $n=2,3,4, \ldots$. We know that the radius of convergence of the Dirichlet series can be obtained, and our computations show that, if $\left|b_{1}\right|<1$, then the series converges absolutely for $z>-\left(\ln \left(\lim \left|\frac{b_{n}}{b_{n+1}}\right|\right)-\ln \left|B_{2}\right|\right)$. Detailed convergence criterion of the above series can be found in Riesz [21]. An important physical quantity of interest is the skin friction coefficient $H^{\prime \prime}(0)$ which is defined as

$$
H^{\prime \prime}(0)=6 B_{1}^{3} \sum_{n=1}^{\infty} n^{2} b_{n} B_{2}^{n}
$$

Furthermore, we are now in the process of finding the two unknown constants $B_{1}$ and $B_{2}$. For this, we make use of other two initial conditions in (22) namely:

$$
\begin{aligned}
& H(0)=\frac{B_{1}^{2}-M^{* 2}}{B_{1}}+6 B_{1} \sum_{n=1}^{\infty} b_{n} B_{2}^{n}=0, \\
& H^{\prime}(0)=-6 B_{1}^{2} \sum_{n=1}^{\infty} n b_{n} B_{2}^{n}=-1 .
\end{aligned}
$$

With few terms in the Dirichlet series, we use Newton's method for nonlinear system of equations to determine these unknown parameters up to required accuracy for all values of $\beta$ and $M^{*}$. The obtained results are compared with those produced by the numerical solution of the boundary value problem (21) and (22) and are presented in Table 3. From this table, the skin friction value $H^{\prime \prime}(0)$ compares well with that of numerical solution for all values of $\beta$ and $M^{*}$. Once the constants $B_{1}$ and $B_{2}$ are determined, it is important to investigate the behavior of the velocity profiles for different values of pressure gradient parameter $\beta$ and $M^{*}$. Note that, the direct quantitative comparison of solution by the Dirichlet series with that of solution obtained in the previous section is almost not possible, since the numerical value for parameter $\lambda$ used in the former method completely disappears in the latter case, and also exact value for $\lambda$ cannot be predicted because the system (21) and (22) is derived in the limit of asymptotically large $\lambda$.

On the other hand, for $\lambda \rightarrow-\infty$, the MHD Falkner-Skan problem (6) and (7) under transformations $f(\eta)=(-\lambda)^{1 / 2} H(z)$, $\eta=(-\lambda)^{-1 / 2} z$ and $M=(-\lambda)^{1 / 2} M^{*}$, we still get the same Eq. (21) but with following different boundary conditions:

$$
H(0)=0, \quad H^{\prime}(0)=1, \quad H^{\prime}(\infty)=0 .
$$

Thus, following a similar procedure as discussed before, the asymptotic problem (21) along with modified boundary conditions (30) has been solved using the Dirichlet series, and results presented in Table 4.

\subsection{Large $\eta$ asymptote: far field behavior}

In the present section, we give the asymptotic expansion of large $\eta$ for the MHD Falkner-Skan equation. Thus, it is enough to study large $\eta$ behavior around $\left|f^{\prime}(\eta)-1\right| \ll 1$ as $\eta \rightarrow \infty$. Therefore, we write

$$
f(\eta) \sim \eta+E(\eta)
$$

which satisfies the derivative condition at infinity, and it is assumed that $E^{\prime}(\eta) \ll 1$. Also, it is instructive to compare the solutions of (31) with those of our exact solutions for all values. Substituting (31) into the MHD Falkner-Skan system (6) and (7), and upon linearizing the resulting ordinary differential equation, we get

$$
E^{\prime \prime \prime}(\eta)+\eta E^{\prime \prime}(\eta)+\left(2 \beta+M^{2}\right) E^{\prime}(\eta)=0, \quad{ }^{\prime}=\frac{d}{d \eta}
$$

and boundary conditions take the form

$$
E(0)=0, \quad E^{\prime}(0)=-(1+\lambda), \quad E^{\prime}(+\infty)=0 .
$$

Solution of Eq. (32) subjected to the conditions (33) is given by

$$
E^{\prime}(\eta)=-(1+\lambda) F\left(-\beta-\frac{M^{2}}{2}, \frac{1}{2},-\frac{\eta^{2}}{2}\right)+(1+\lambda) \frac{W}{2 \sqrt{2}} \eta F\left(\frac{1-2 \beta-M^{2}}{2}, \frac{3}{2},-\frac{\eta^{2}}{2}\right)
$$

where $W=\frac{\Gamma(1 / 2)}{\Gamma(3 / 2)} \frac{\Gamma\left(1+2 \beta+\frac{M^{2}}{2}\right)}{\Gamma\left(\frac{1}{2}+\beta+\frac{M^{2}}{2}\right)}, \Gamma$ is the Gamma function and $F(\widetilde{a}, \widetilde{b}, \eta)$ is the confluent hypergeometric function. The solution in terms of $f(\eta)$ is given by $f^{\prime}(\eta)=1+E^{\prime}(\eta)$ The skin friction is given by

$$
f^{\prime \prime}(0)=\frac{W(1+\lambda)}{\sqrt[2]{2}} .
$$


Table 3 compares the values of skin friction $f^{\prime \prime}(0)$ obtained by exact solution in Section 3 and with those given by (35). The results are quite remarkable.

\section{Results and discussion}

In this paper, we have developed an exact solution of the MHD Falkner-Skan equation for general values of pressure gradient parameter $\beta$ and magnetic parameter $M$. The known exact solution for $\beta=-1$ and $M=0$ which is in terms of error and exponential functions, has been exploited and rewritten, for convenience, to obtain exact solution for general values of $\beta$ and $M$. The present method embeds the known exact solution as a special case. We have presented large $\lambda$ and $\eta$ asymptotics for the problem in discussion, and obtained corresponding solutions. The Dirichlet series solution is particularly useful when the derivative boundary condition at infinity is zero. In any case, representative results for the velocity profiles $\left(f^{\prime}(\eta)\right)$ and skin friction coefficient $\left(f^{\prime \prime}(0)\right.$ and $\left.H^{\prime \prime}(0)\right)$ have been obtained by assigning the numerical values to the different parameters ( $\beta, M$ and $\lambda$ ) encountered in the problem. Again, in order to verify our results and accuracy of the presented solutions, we have compared our results for the skin friction coefficient $f^{\prime \prime}(0)$ with those produced by the DNS of the problem. Also, to validate our analytical results and to understand the physical behavior, we present the velocity profiles in Figs. 1-6, and skin friction coefficient in Tables $1-4$.

Ranges of parameter space which proved to be the most interesting from the physical dynamics of the problem have been investigated through the velocity profiles and studying their behavior as a function of coordinate distance.

Fig. 1 describes the velocity profiles $f^{\prime}(\eta)$ as a function of $\eta$ for an exact solution for $\beta=-1$ and $M=0$ for different wall stretching parameter $\lambda$. It is clearly seen that all velocity curves asymptotically satisfy their derivative condition at infinity (i.e. $f^{\prime}(\eta) \rightarrow 1$ as $\eta \rightarrow \infty$ ). It is also observed that the velocity curves first increase to peak level for some distance and start to decrease as they move along the boundary layer. However, the velocity curves in Fig. 2 behave differently when we plotted

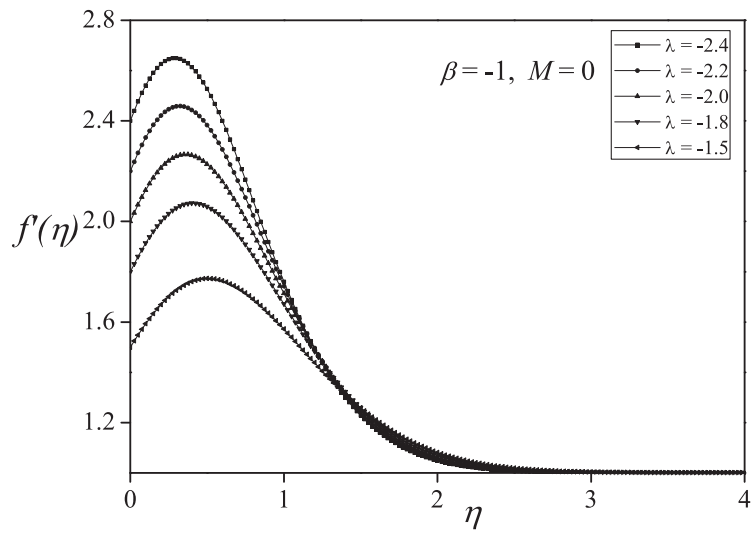

Fig. 1. Variation of velocity profiles $f^{\prime}(\eta)$ with $\eta$ for $\beta=-1$ and $M=0$ for different values of $\lambda$.

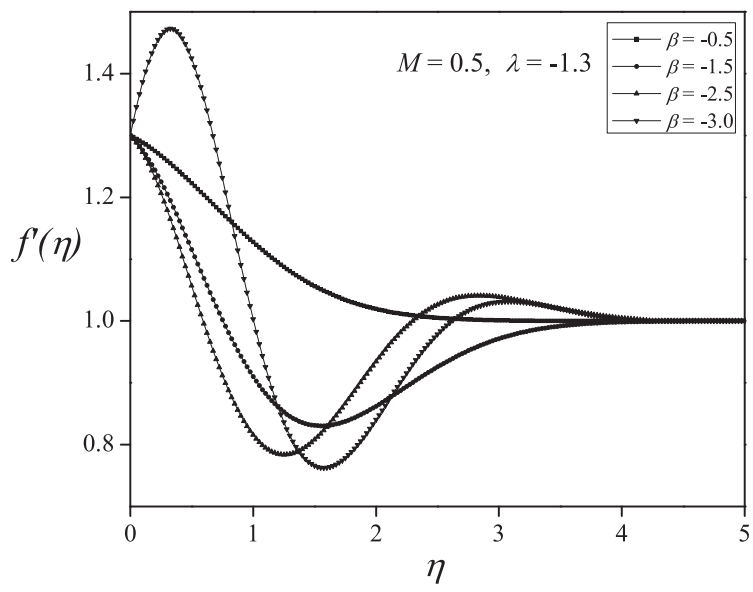

Fig. 2. Variation of velocity profiles $f^{\prime}(\eta)$ with $\eta$ for different values of pressure gradient parameter $\beta<0$ with Hartman number $M=0.5$. Note that $f^{\prime}(0)=-\lambda$. 


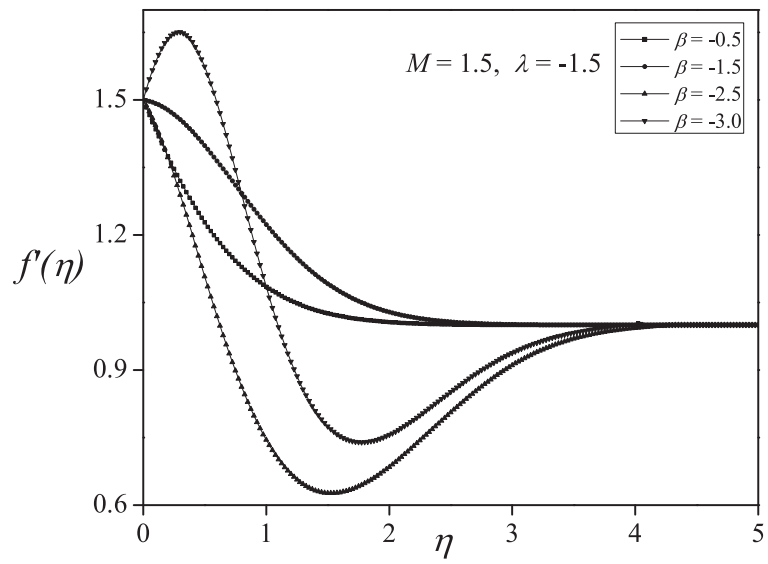

Fig. 3. Variation of velocity profiles $f^{\prime}(\eta)$ with $\eta$ for different values of pressure gradient parameter $\beta<0$ with Hartmann number $M=1.5$. Note that $f^{\prime}(0)=-\lambda$.

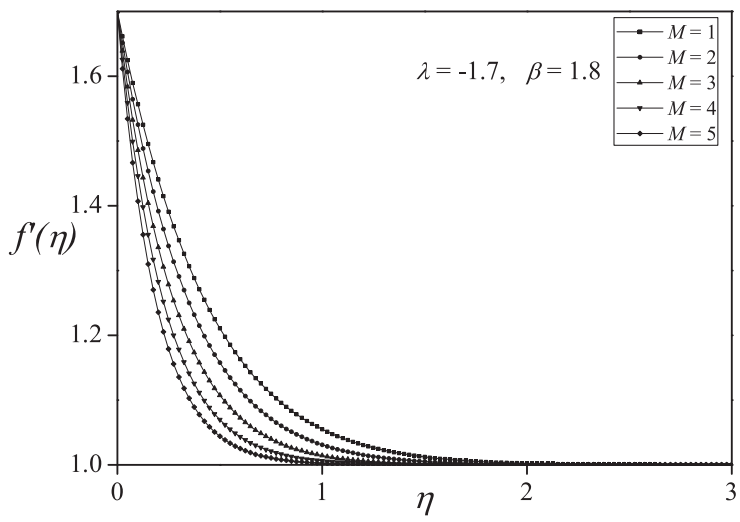

Fig. 4. Variation of velocity profiles $f^{\prime}(\eta)$ with $\eta$ for different values of magnetic parameter $M$ with $\beta=1.8$. Note that $f^{\prime}(0)=-\lambda$.

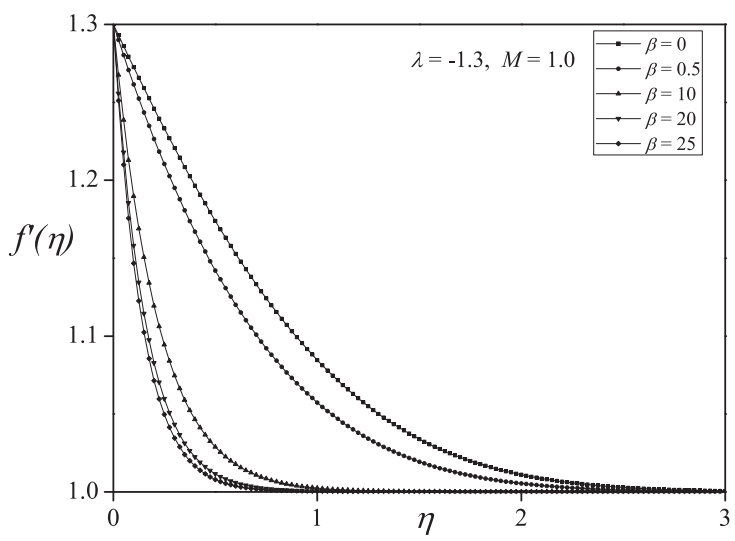

Fig. 5. Variation of velocity profiles $f^{\prime}(\eta)$ with $\eta$ for different values of $\beta>0$ with a Hartman number $M=1.0$. Note that $f^{\prime}(0)=-\lambda$.

them for different values of decelerated flow parameter $\beta(<0)$ for fixed $\lambda=-1$.3. For all values of $\beta$ investigated except $\beta=-2.5$, all the curves first decrease and cross their boundary region which represents undershoot $\left(f^{\prime}(\eta)<1\right.$ for some $\left.\eta\right)$ but eventually, they satisfy their end condition. Whereas for $\beta=-2.5$, surprisingly, the curve experiences both overshoot $\left(f^{\prime}(\eta)>1\right.$ for some $\left.\eta\right)$ first to attain a maximum value and it undershoots suddenly, but, at the end it satisfies end condition. These curves are similar to oscillations however far from the standard trigonometric oscillations. As pointed out by Hastings 


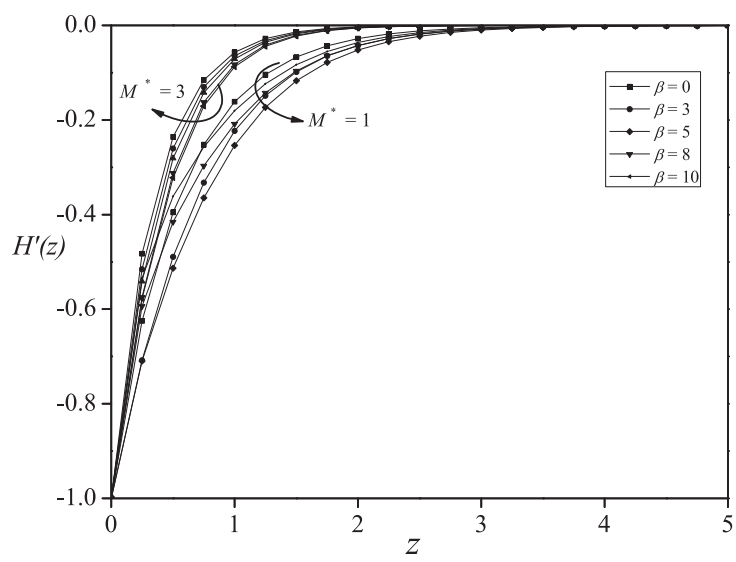

Fig. 6. Variation of velocity profiles $H^{\prime}(z)$ with $z$ for different values of $\beta$ for two values of $M^{*}$. All the curves are obtained by the Dirichlet series solution (27).

Table 1

Comparison of the results of skin friction $f^{\prime \prime}(0)$ obtained by an exact solution (11) and (15) with the DNS of the MHD Falkner-Skan equation.

\begin{tabular}{|c|c|c|c|c|c|c|c|c|}
\hline \multicolumn{3}{|c|}{$\beta=0.5$} & \multicolumn{3}{|c|}{$\beta=1.2$} & \multicolumn{3}{|c|}{$\beta=2.2$} \\
\hline$\lambda$ & $\begin{array}{l}\text { Analytical } \\
\text { solution }\end{array}$ & $\begin{array}{l}\text { Numerical } \\
\text { solution }\end{array}$ & $\lambda$ & $\begin{array}{l}\text { Analytical } \\
\text { solution }\end{array}$ & $\begin{array}{l}\text { Numerical } \\
\text { solution }\end{array}$ & $\lambda$ & $\begin{array}{l}\text { Analytical } \\
\text { solution }\end{array}$ & $\begin{array}{l}\text { Numerical } \\
\text { solution }\end{array}$ \\
\hline \multicolumn{9}{|c|}{$M=0.5$} \\
\hline-1.2 & -0.279478 & -0.27949 & -1.2 & -0.36887 & -0.36971 & -1.2 & -0.47049 & -0.47049 \\
\hline-1.4 & -0.578654 & -0.57866 & -1.4 & -0.76417 & -0.76379 & -1.4 & -0.97321 & -0.9711 \\
\hline-1.8 & -1.2277 & -1.23213 & -1.8 & -1.62035 & -1.62057 & -1.8 & -2.05668 & -2.05722 \\
\hline-2.3 & -2.14503 & -2.14417 & -2.3 & -2.81037 & -2.81048 & -2.3 & -3.56228 & -3.56236 \\
\hline-2.5 & -2.53658 & -2.53654 & -2.5 & -3.33078 & -3.32096 & -2.5 & -4.2091 & -4.20731 \\
\hline-2.8 & -2.53349 & -2.53654 & -2.8 & -4.1237 & -4.12162 & -2.8 & -5.19228 & -5.21817 \\
\hline-3 & -3.59277 & -3.58162 & -3 & -4.6778 & -4.67786 & -3 & -5.91903 & -5.92007 \\
\hline \multicolumn{9}{|l|}{$M=1$} \\
\hline-1.2 & -0.32776 & -0.32774 & -1.2 & -0.40794 & -0.40793 & -1.2 & -0.50125 & -0.50124 \\
\hline-1.4 & -0.67318 & -0.67222 & -1.4 & -0.83807 & -0.838 & -1.4 & -1.03081 & -1.03082 \\
\hline-1.8 & -1.40866 & -1.40906 & -1.8 & -1.7611 & -1.76112 & -1.8 & -2.16634 & -2.1704 \\
\hline-2.3 & -2.41444 & -2.41443 & -2.3 & -3.02554 & -3.02554 & -2.3 & -3.73527 & -3.73558 \\
\hline-2.5 & -2.93242 & -2.84133 & -2.5 & -3.57451 & -3.56369 & -2.5 & -4.40299 & -4.40284 \\
\hline-2.8 & -3.50702 & -3.50701 & -2.8 & -4.40288 & -4.40391 & -2.8 & -5.44559 & -5.44562 \\
\hline-3 & -3.96754 & -3.96716 & -3 & -4.97537 & -4.98537 & -3 & -6.16656 & -6.16785 \\
\hline
\end{tabular}

Table 2

Comparison of the results of skin friction $f^{\prime \prime}(0)$ obtained by an exact solution (11) and (15) with asymptotic solution (26) of the MHD Falkner-Skan equation in the limit of large $\eta$.

\begin{tabular}{|c|c|c|c|c|c|c|c|c|}
\hline \multicolumn{3}{|c|}{$\beta=0.5$} & \multicolumn{3}{|c|}{$\beta=1.2$} & \multicolumn{3}{|c|}{$\beta=2.2$} \\
\hline$\lambda$ & $\begin{array}{l}\text { Analytical } \\
\text { solution }\end{array}$ & $\begin{array}{l}\text { Asymptotic } \\
\text { solution }\end{array}$ & $\lambda$ & $\begin{array}{l}\text { Analytical } \\
\text { solution }\end{array}$ & $\begin{array}{l}\text { Asymptotic } \\
\text { solution }\end{array}$ & $\lambda$ & $\begin{array}{l}\text { Analytical } \\
\text { solution }\end{array}$ & $\begin{array}{l}\text { Asymptotic } \\
\text { solution }\end{array}$ \\
\hline \multicolumn{9}{|c|}{$M=0.8$} \\
\hline-1.2 & -0.30464 & -0.29619 & -1.2 & -0.39011 & -0.3781 & -1.2 & -0.48771 & -0.471685 \\
\hline-1.4 & -0.62883 & -0.59237 & -1.4 & -0.80332 & -0.75619 & -1.4 & -1.00344 & -0.943371 \\
\hline-1.8 & -1.32675 & -1.18474 & -1.8 & -1.69515 & -1.51239 & -1.8 & -2.11664 & -1.88674 \\
\hline-2.3 & -2.2882 & -1.92521 & -2.3 & -2.92415 & -2.45763 & -2.3 & -3.6544 & -3.06595 \\
\hline-2.5 & -2.69876 & -2.22139 & -2.5 & -3.4482 & -2.83573 & -2.5 & -4.31013 & -3.53764 \\
\hline-2.8 & -3.3422 & -2.66567 & -2.8 & -4.27144 & -3.40287 & -2.8 & -5.33755 & -4.24517 \\
\hline-3 & -3.78655 & -2.96186 & -3 & -4.84121 & -3.78097 & -3 & -6.05009 & -4.24517 \\
\hline
\end{tabular}

and Troy [22] for classical Falkner-Skan equation, solutions in the current problem for MHD Falkner-Skan flow also oscillate a finite number of times, but eventually tend to the prescribed boundary condition. This typical phenomenon is true even for increasing magnetic parameter $M$ and $\lambda$ (absolute) which is shown in Fig. 3.

The effects of magnetic parameter $M$ on horizontal velocity $f^{\prime}(\eta)$ are shown in Fig. 4 when other fixed parameters held constant $(\beta>0)$. It is observed that the velocity monotonically tends to 1 when the distance moves along the boundary. Also, 
Table 3

Comparison of the results for skin friction $H^{\prime \prime}(0)$ obtained by the Dirichlet series (27) with numerical solution of asymptotic problem (21) and (22).

\begin{tabular}{|c|c|c|c|c|c|c|c|c|}
\hline \multirow[t]{2}{*}{$M^{*}$} & \multicolumn{4}{|l|}{$\beta=0$} & \multicolumn{4}{|l|}{$\beta=0.5$} \\
\hline & $B_{2}$ & $B_{1}$ & Dirichlet & Numerical & $B_{2}$ & $B_{1}$ & Dirichlet & Numerical \\
\hline 2 & 0.0498 & 1.7452 & 1.91352 & 1.91689 & 0.0524 & 1.7390 & 1.82609 & 1.82688 \\
\hline 3 & 0.0199 & 2.8318 & 2.94406 & 2.94448 & 0.0203 & 2.8301 & 2.88695 & 2.88707 \\
\hline 4 & 0.0108 & 3.8743 & 3.95825 & 3.95837 & 0.0109 & 3.8736 & 3.91588 & 3.91591 \\
\hline \multirow[t]{3}{*}{5} & 0.0068 & 4.8996 & 4.96664 & 4.96668 & 0.0068 & 4.8993 & 4.93293 & 4.93295 \\
\hline & \multicolumn{4}{|l|}{$\beta=1.5$} & \multicolumn{4}{|l|}{$\beta=2.5$} \\
\hline & $B_{2}$ & $B_{1}$ & Dirichlet & Numerical & $B_{2}$ & $B_{1}$ & Dirichlet & Numerical \\
\hline 2 & 0.0591 & 1.7241 & 1.63168 & 1.63152 & 0.0682 & 1.7054 & 1.42205 & 1.40808 \\
\hline 3 & 0.0212 & 2.8266 & 2.76857 & 2.76848 & 0.0222 & 2.8227 & 2.64553 & 2.6445 \\
\hline 4 & 0.0112 & 3.8722 & 3.82959 & 3.82957 & 0.0115 & 3.8707 & 3.74143 & 3.74124 \\
\hline 5 & 0.0069 & 4.8986 & 4.86478 & 4.86476 & 0.00709 & 4.8979 & 4.79569 & 4.79557 \\
\hline
\end{tabular}

Table 4

Comparison of the results for skin friction $H^{\prime \prime}(0)$ obtained by the Dirichlet series (27) with numerical solution of asymptotic problem (21) and (30).

\begin{tabular}{|c|c|c|c|c|c|c|c|c|}
\hline \multirow[t]{2}{*}{$M^{*}$} & \multicolumn{4}{|l|}{$\beta=0$} & \multicolumn{4}{|l|}{$\beta=0.5$} \\
\hline & $B_{2}$ & $B_{1}$ & Dirichlet & Numerical & $B_{2}$ & $B_{1}$ & Dirichlet & Numerical \\
\hline 2 & -0.03563 & 2.24446 & -2.08321 & -2.08316 & -0.03443 & 2.24012 & -2.16095 & -2.16093 \\
\hline 3 & -0.01723 & 3.16507 & -3.05554 & -3.05551 & -0.01694 & 3.16365 & -3.10936 & -3.10935 \\
\hline 4 & -0.00999 & 4.12434 & -4.04166 & -4.04161 & -0.00989 & 4.12371 & -4.08259 & -4.08256 \\
\hline \multirow[t]{3}{*}{5} & -0.00649 & 5.09966 & -5.03333 & -5.03324 & -0.00645 & 5.09934 & -5.06628 & -5.06623 \\
\hline & \multicolumn{4}{|l|}{$\beta=1.5$} & \multicolumn{4}{|l|}{$\beta=2.5$} \\
\hline & $B_{2}$ & $B_{1}$ & Dirichlet & Numerical & $B_{2}$ & $B_{1}$ & Dirichlet & Numerical \\
\hline 2 & -0.03231 & 2.23226 & -2.30877 & -2.30883 & -0.03049 & 2.22535 & -2.44613 & -2.44796 \\
\hline 3 & -0.01640 & 3.16095 & -3.21433 & -3.21435 & -0.01589 & 3.15841 & -3.31603 & -3.31607 \\
\hline 4 & -0.00971 & 4.12251 & -4.16323 & -4.16326 & -0.00952 & 4.12135 & -4.24236 & -4.24245 \\
\hline 5 & -0.00636 & 5.0987 & -5.13155 & -5.13155 & -0.00629 & 5.09809 & -5.19600 & -5.19601 \\
\hline
\end{tabular}

velocity decreases for increasing values of magnetic parameter. Furthermore, the momentum boundary layer thickness decreases as magnetic parameter $M$ increases. This is due to the fact that as $M$ increases, the boundary layer flow acquires more magnetization that leads to the variation in Lorentz force which opposes the flow. Finally, we should also point out explicitly that the presence of a magnetic field in the model has both qualitative and quantitative effects on the boundary layer flow compared to the results for $M=0$ (Sachdev et al. [8]). Fig. 5 illustrates the effects of pressure gradient parameter $\beta$ on the velocity profiles when other parameters held constant. Here for $\beta=0$ and $M=0$ case represents the flow on the flat plate which is the famous Blasius equation and hence no comments are made. It is observed that as analogous to results in the previous discussion, pressure gradient parameter $\beta$ increases the boundary layer thickness. In these figure, it is observed that all curves experience neither overshoot or undershoot for these parameters.

Table 1 compares the values for the skin-friction coefficient $f^{\prime \prime}(0)$ obtained by exact solution with that of the DNS of the problem for various values of $\beta$ and $M$. We see that there is an excellent agreement between two solutions for all values presented in table. Also as the wall stretching parameter increases (in absolute sense), skin-friction coefficient also increases. Also, as both $\beta$ and $M$ increase, it again increases. Comparison of the skin-friction coefficient obtained by solving linearized version of the problem for large $\eta$ asymptotics (Section 4.2) with that of solution obtained by our exact method (Section 3 ) is presented in Table 2 for various values of $\beta$. It is that the results are just accurate as the results of linearized differential equation deviate away from the exact solution, however the physical intuition can be drawn from the results. If we allow the solutions of the linear equation to use for further analyses (to compute the velocity profiles or skin friction, etc.), eventually the results of the linear theory will become invalid, because the results are not accurate enough especially for large $\beta$ and $M$. Then solution will be defined by the nonlinearities in the MHD Falkner-Skan equation. Therefore, we should rely on the most accurate exact solution method such as the method developed in the previous section (Section 3 ). This difference between these solutions becomes clear upon inspecting the results in Table 2.

Nevertheless, in contrast to the results of Table 2, we present results of the asymptotic solution for the case of $\lambda \rightarrow+\infty$ in Table 3 and that of $\lambda \rightarrow-\infty$ in Table 4, the results of the Dirichlet series are remarkably in good agreement with the DNS of the asymptotic problem. In fact we could compare the above results with that of exact solutions obtained for general $\beta$ and $M$ by new method presented in Section 3 in the limit of large $\lambda$, but in the system (21), (22) and (21), (30), the parameter $\lambda$ disappears explicitly. Instead we have compared the results with the DNS of the problem as our exact solution method also compares equally well with numerical solutions. Further, Fig. (6) depicts the variation of horizontal velocity profiles $H^{\prime}(z)$ for different values of pressure gradient parameter $\beta$ for two values of $M^{*}$. We use the Dirichlet series solution (23) to plot these 
curves. It is observed that the effects of pressure gradient is decrease thickness of the boundary layer. Again, boundary layer thickness decreases further for increasing values of $M^{*}$. Therefore, we anticipate that the effects of $\beta$ and $M^{*}$ are to decrease the velocity as a result momentum boundary layer thickness reduces.

\section{Conclusions}

We have developed a new exact method for the solution of MHD Falkner-Skan equation which describes a two-dimensional MHD boundary layer flow over a semi-infinite flat plate in the presence of a uniform magnetic field. This equation exhibits a closed form solution only for $\beta=-1$ and $M=0$. This closed form solution has been exploited to obtained an exact solution of the problem for all values of $\beta$ and $M$. Further, two asymptotic solutions in the limit of large $\lambda$ and $\eta$, also have been obtained and solved for various physical parameters. The Dirichlet series is particularly useful when one of the boundary conditions at infinity is zero. In any case, all the results thus obtained are compared with DNS of the problem, and found that the results agree quite well up to required accuracy. Further, it is shown that the effects of pressure gradient $\beta$ and magnetic parameter $M$ are to decrease the velocity and to decrease the momentum boundary layer thickness.

\section{Acknowledgements}

Two authors RBK and NMB are grateful to the University Grants Commission, New Delhi, India, for providing financial support under a major research project Grant No. 39-32/2010 (SR) to carry out work. Authors would like to thank referees for the valuable comments which enhanced the quality of the paper.

\section{References}

[1] Kumaran V, Banerjee AK, Vanav Kumar A, Vajravelu K. MHD flow past a stretching permeable sheet. Appl Math Comput 2009;210:26-32.

[2] Joneidi AA, Domairry G, Babaelahi M. Analytical treatment of MHD free convective flow and mass transfer over a stretching sheet with chemical reaction. J Taiwan Inst Chem Eng 2010;41:35-43.

[3] Su X, Zheng L. Approximate solutions to MHD Falkner-Skan flow over permeable wall. Appl Math Mech 2011;32:401-8.

[4] Guedda M, Aly EH, Ouahsine A. Analytical and ChPDM analysis of MHD mixed convection over a vertical flat plate embedded in a porous medium filled with water at 4C. J Appl Math Model 2011;35:5182-97.

[5] Abbasbandy S, Hayat T. Solution of the MHD Falkner-Skan flow by homotopy analysis method. Commun Nonlinear Sci Numer Simul 2009;14:3591-8.

[6] Hendi FA, Hussain M. Analytic solution for MHD Falkner-Skan flow over a pourous surface. J Appl Math 2012. http://dx.doi.org/10.1155/2012/123185.

[7] Parand K, Rezaei AR, Ghaderi SM. An approximate solution of the MHD Falkner-Skan flow by Hermite functions pseudospectral method. Commun Nonlinear Sci Numer Simul 2011;16:274-83.

[8] Sachdev PL, Kudenatti RB, Bujurke NM. Exact analytic solution of a boundary value problem for the Falkner-Skan equation. Stud Appl Math 2008;120:1-16.

[9] Kudenatti RB, Awati VB. Solution of pressure gradient stretching plate with suction. Appl Math Comput 2009;210:151-7.

[10] Kudenatti RB. A new exact solution for boundary layer flow over a stretching plate. Int J Non-Linear Mech 2012;47:727-33.

[11] Fang T. Boundary layer flow over a shrinkingsheet with power-law velocity. Int J Heat Mass Transfer 2008;51:5838-43.

[12] Bachok N, Ishak A, Pop I. Unsteady three-dimensional boundary layer flow due to a permeable shrinking sheet. Appl Math Mech 2010;31:1421-8.

[13] Sajid M, Hayat T. The application of homotopy analysis method for MHD viscous flow due to a shrinking sheet. Chaos, Solitons Fractals 2009;39:1317-23.

[14] Hayat T, Javed T, Sajid M. Analytic solution for MHD rotating flow of a second grade fluid over a shrinking surface. Phys Lett A 2008;372:3264-73.

[15] Das K. Slip effects on MHD mixed convection stagnation point flow of a micropolar fluid towards a shrinking vertical sheet. Comput Math Appl 2012;63:255-67.

[16] Nadeem S, Awais M. Thin film flow of an unsteady shrinking sheet through porous medium with variable viscosity. Phys Lett A $2008 ; 372: 4965-72$.

[17] Javed T, Abbas Z, Sajid M, Ali N. Heat transfer analysis for a hydromagnetic viscous fluid over a non-linear shrinking sheet. Int J Heat Mass Transfer 2011;54:2034-42.

[18] Guedda M, Ouahsine A. Similarity solutions of MHD flow in a saturated porous medium. Eur J Mech B Fluids 2012;33:87-94.

[19] Kravnchenko TK, Yablonskii AI. Solution of an infinite boundary value problem for third order equation. Differ Uraneniya 1965;1:327-31.

[20] Kudenatti RB, Awati VB, Bujurke NM. Approximate analytical solutions of a class of boundary layer equations over nonlinear stretching surface. Appl Math Comput 2011;218:2952-9.

[21] Riesz S. Introduction to Dirichler Series. CUP; 1957.

[22] Hastings SP, Troy W. Oscillating solutions of the Falkner-Skan equation for negative. SIAM J Math Anal 1987;18:422-9. 